\section{Dr. Choe and Dr. Kim reply}

\section{To the Editor:}

We thank Drs. Dalbeth and McQueen for their kind comments ${ }^{1}$ on our recent article on radiographic bone damage between chronic gout and inflammatory cytokines ${ }^{2}$. We assessed radiographic damage of the involved foot joints in patients with chronic gout according to the modified Sharp/van der Heijde system validated by Dr. Dalbeth's group ${ }^{3}$. Dr. Dalbeth has expressed skepticism about the low scores for radiographic damage in our patients with gout.

First, we examined all joints in both feet. Eighteen patients among the enrolled patients had radiographic damage such as joint space narrowing and bony erosion. We found that the mean total damage scores in the involved joints were $3.5 \pm 2.0 \mathrm{SD}$. The data presented in Table 1 are from both those who had radiographic damage and those who had no radiographic damage. We are sorry that the data in Table 1 were not clear and were misunderstood by Dr. Dalbeth. There are several possible reasons for the lower than expected radiographic scores in our study. The enrolled patients had relatively mild severity that was well controlled. There might also have been a selection bias in the patient enrollment. In addition, there is the possibility that we underestimated radiographic damage.

We fully agree that both bone damage and its radiographic assessment in gout are crucial issues. Dr. Dalbeth's comments will be valuable to our future research in gout. We feel that the clinical significance of these issues should be reassessed in a larger population with variable clinical stages of gout.

JUNG-YOON CHOE, MD, PhD; SEONG-KYU KIM, MD, PhD, Department of Internal Medicine, Arthritis and Autoimmunity Research Center, Catholic University of Daegu School of Medicine, Daegu, Republic of Korea. Address correspondence to Dr. S-K. Kim, Department of Internal Medicine, Arthritis and Autoimmunity Research Center, Catholic University of Daegu School of Medicine, 3056-6 Daemyung 4-Dong, Namgu, Daegu 705-718, Republic of Korea. E-mail: kimsk714@cu.ac.kr

\section{REFERENCES}

1. Dalbeth N, McQueen FM. Clarification of the modified radiographic damage scoring method for gout [letter]. J Rheumatol 2012;39:874.

2. Choe JY, Lee GH, Kim SK. Radiographic bone damage in chronic gout is negatively associated with the inflammatory cytokines soluble interleukin 6 receptor and osteoprotegerin. J Rheumatol 2011;38:485-91.

3. Dalbeth N, Clark B, McQueen F, Doyle A, Taylor W. Validation of a radiographic damage index in chronic gout. Arthritis Rheum 2007;57:1067-73.

J Rheumatol 2012;39:4; doi:10.3899/jrheum.111280 